\title{
Robust Memristor Networks for Neuromorphic Computation Applications
}

\author{
Dániel Hajtó ${ }^{1, *}$ and Ádám Rák ${ }^{2}$ and György Cserey ${ }^{1,2}$ (I) \\ 1 Faculty of Information Technology and Bionics, Pázmány Péter Catholic University, 1083 Budapest, Hungary; \\ cserey.gyorgy@itk.ppke.hu \\ 2 StreamNovation Ltd., 1083 Budapest, Hungary; streamnovation@streamnovation.com \\ * Correspondence: hajto.daniel@itk.ppke.hu
}

Received: 30 September 2019; Accepted: 25 October 2019; Published: 31 October 2019

check for updates

\begin{abstract}
One of the main obstacles for memristors to become commonly used in electrical engineering and in the field of artificial intelligence is the unreliability of physical implementations. A non-uniform range of resistance, low mass-production yield and high fault probability during operation are disadvantages of the current memristor technologies. In this article, the authors offer a solution for these problems with a circuit design, which consists of many memristors with a high operational variance that can form a more robust single memristor. The proposition is confirmed by physical device measurements, by gaining similar results as in previous simulations. These results can lead to more stable devices, which are a necessity for neuromorphic computation, artificial intelligence and neural network applications.
\end{abstract}

Keywords: memristor; neuromorphic computing; artificial intelligence; hardware-based deep learning ICs; circuit design

\section{Introduction}

Since the theoretical [1] and practical [2] discovery of memristors, they have been extensively studied [3-5] as elementary building blocks for artificial intelligence and neuromorphic computing applications.

The expected properties of memristors for such applications are wide and analog resistance range, low variance of device parameters and high device stability during long-term operation. Research has been done [6] to find optimal materials that satisfy these expectations, but even then there are other possibilities to further increase the capabilities of memristors.

In binary memory applications, three important properties should be considered. The first one is having two clearly distinguishable states and these state declarations should apply to every element in a memory array. The second one is having a fast switching speed between the states. To reach the performance of the current complementary metal-oxide-semiconductor (CMOS) technology's RAM the switching speed should be less than $10 \mathrm{~ns}$. The third one is cycle endurance, which is the number of write-erase cycles without permanent device failure.

In crossbar-network applications, a certain amount of uniformity of the memristors is necessary. The programming voltage and current levels are the same for every element and thus one expects that they will behave similarly for the same input signals.

In the case of ANN applications, more deviance could be tolerated, but many state devices are needed, so the memristors developed for binary or multi-state memory purposes will not be sufficient.

The mass production of devices, which can reliably fulfill these requirements, is not trivial. If the production yield of single devices is less than 100 percent (as they are not functioning 
as memristors or they are outside of the accepted range of parameters), then they can also affect the access circuit and the encompassing parts of the neuromorphic system.

If the production yield of single devices is less than 100 percent (as they are not functioning as memristors or they are outside of the accepted range of parameters), then they can also affect the access circuit and the encompassing parts of the neuromorphic system.

In very large scale integration (VLSI) device manufacturing, it is often easier and tends to cause fewer faults to make the same device many times, and use it as a building block to emulate other devices, instead of creating fewer, but different devices [7]. The same approach can be applied to memristors, but one should take into consideration their special nonlinear behavior in the voltage-current domain. This idea is further supported by the fact that memristors as two-terminals, could be manufactured more easily on many layers on microchips [8] than transistors. However, with every extra layer, the probability of device defects could also increase.

In order to maintain or even improve the virtual yield of the production, interconnected structures of the memristor network are proposed. These circuits and the presented measurement results provide a response to the above mentioned challenges. Our proposed circuit constructions can be efficiently implemented on microchips, stacking the memristors of the circuit on top of each other. If a decent multilayer production technology arises with memristors, the disadvantage of the usage of several layers for the implementation of a single layer of memristor would be neglectable.

This paper is organized as follows: after the above problem proposal, the measurement environment is introduced and explanatory discussion is given about our circuitry. The third section contains the proposed circuits and the measurement results that are more detrimental to the yield. This circuitry effectively addresses the proposed task. In the fourth section, the results are summarized and analyzed. The article is closed with a brief summary of the results in the conclusion section.

\section{Materials and Methods}

\subsection{Materials}

The measured memristor devices are made of $\mathrm{Ge}_{2} \mathrm{Se}_{3}$ (germanium-selenide) and $A g$ (silver) based chalcogenide dielectric with $W$ (Tungsten) conductors. The devices have a switching threshold, meaning that under a certain threshold voltage $(0.1 \mathrm{~V}$ in our case), their state does not change. This feature makes the memristor implementation desirable for applications where reading the state should not change the state itself. On the other hand, usually it has very few metallic dendrites, which makes the characteristic very coarse. The memristors are current-controlled and the typical writing-erasing voltages are $2.5 \mathrm{~V}$. One of the consequences of being current-controlled is that the erasing process is faster than the writing process.

The measurement setup consists of an amplifier circuit as a current-voltage converter and a current regulator resistor as it can be seen in Figure 1. The current regulator resistor helped to ensure that the current does not reach high values where the device could become faulty. The used signal generator and measurement device is an "NI ELVIS II+", controlled by LabView software (National Instruments, Austin, TX, United States). The sampling frequency is $500 \mathrm{kSample} / \mathrm{s}$ for every measurement. The state of every device has been set to an OFF state before every measurement. 


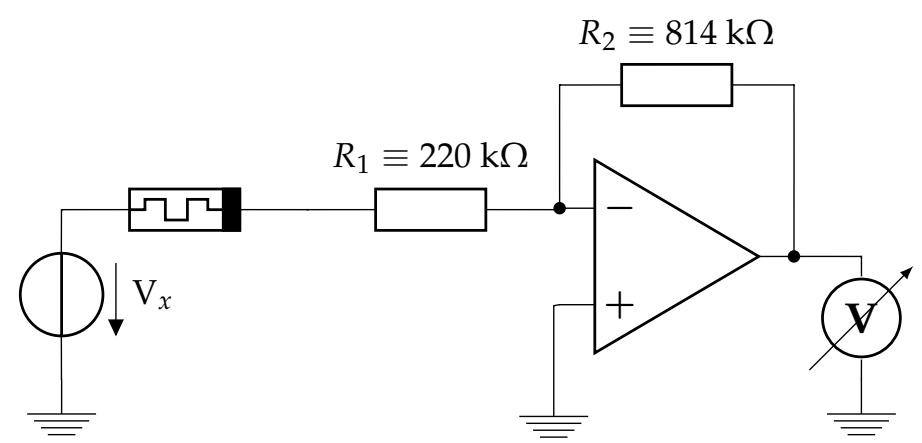

Figure 1. Measurement environment circuit. The measurement setup consists an amplifier circuit as a current controlled voltage source and a current regulator resistor. The used amplifier is a "TL082". The applied voltage $\mathrm{V}_{x}$ was strictly between $-2.5 \mathrm{~V}$ and $2.5 \mathrm{~V}$. The memristor symbol represents either a single memristor or a network of memristors depending on the measurement.

\subsection{Methods}

\subsubsection{Metrics}

First of all, it is important to differentiate two main types of memristors from a functional point of view. The first type is the analog purpose memristor (APM). It operates in the continuous domain, which means it can have any resistance (or conductance) value in its operational range. This might sound unrealistic as we know that at a very low scale, energy levels are quantized, but it can be interpreted as the memristor having so many states that can be considered as infinitely many. Another formal definition is that an APM can store any real value between the normalized range of zero and one.

The second type is the digital (or discrete) purpose memristor (DPM), which has several but countable states and the resistance value can only be one of these states. An important property is that these states should be clearly distinguishable from each other. This type can be used trivially as an $n$ state memory unit based on the number of its possible states.

An extreme, but important case of the DPM is when only two states can be clearly distinguished, as they can be further classified as binary purpose memristors (BPM). With its reduced capabilities they lack applications beside their use as binary memory units supplementary to the CMOS based digital systems or implementing routing in logic gate arrays, like Field programmable gate arrays (FPGAs) [9].

In general, the mass production of BPMs is solved, there are manufacturers [10], who sell commercial devices for an affordable price. DPMs are existing in an early development state at research institutes [11]. APMs, which have practically an infinitely many numbers of states, are yet to be introduced and might even be impossible to produce due to physical limitations [12]; or it requires new quantum mechanical solutions, which are also under development [13]. In general, from an application point of view, digital memory technologies use BPMs, artificial neural networks need at least DPM complexity, and neuromorphic computation applications require APMs.

Our previously introduced circuit proposals [14] were intended to convert several DPMs into a single APM. This was tested through simulations, which showed that this circuit topology can achieve analog behavior when made from solely multi-state memristors. However, in this work real device measurement results are given, which proves that the same circuit can effectively convert several unreliable BPMs into a more reliable one.

The same control signal should produce the same result, both in the transient characteristics and the final state of the memristor. By reliability, we mean a low variance of the characteristics. Our aim was to avoid using a memristor model as an absolute reference, and be able to approximate the real memristors more accurately. Therefore, our analysis focuses on the mean and variance of characteristics of several measurements on the same device or network in a short period of time. 
The index of dispersion has been used as the measure of unreliability. It formulates as the sum of the variance of the signal, normalized by the amplitude of the signal, due to the expectation that higher amplitude signals have naturally higher variance. This measure is valid only for positive data points. For this reason, the absolute value of the signal has been used:

$$
u=\sum_{i}^{N} \frac{\sigma_{i}^{2}}{\left|\mu_{i}\right|}
$$

where $u$ is the unreliability of the device, $N$ is the number of measurement points, $i$ is a measurement point of the measuring signal, $\sigma_{i}^{2}$ is the variance of a measurement point over the consecutive measurements and $\mu_{i}$ is the mean of a measurement point over the consecutive measurements.

The approximation of the yield of a production technology is highly dependent on the available number of samples of the given device. having a limited number of devices, this question can not be addressed, but it has been shown in a previous work [14] that the yield of a production technology can be increased with this method.

The planning and execution of the measurements have been carried out with consideration of previous related studies [15] on memristor measuring techniques.

\subsubsection{Circuits}

The measurements were carried out on four different memristor network circuit topologies of which two were introduced before [14] with corresponding simulation results. The H-fractal (Figure 2a) and checkerboard-like (Figure $2 \mathrm{~b}$ ) topology both gave comparably good results, which shows that verifying both cases with measurements is reasonable. During simulations with heavy defect probability, the checkerboard-like topology has given slightly better results.

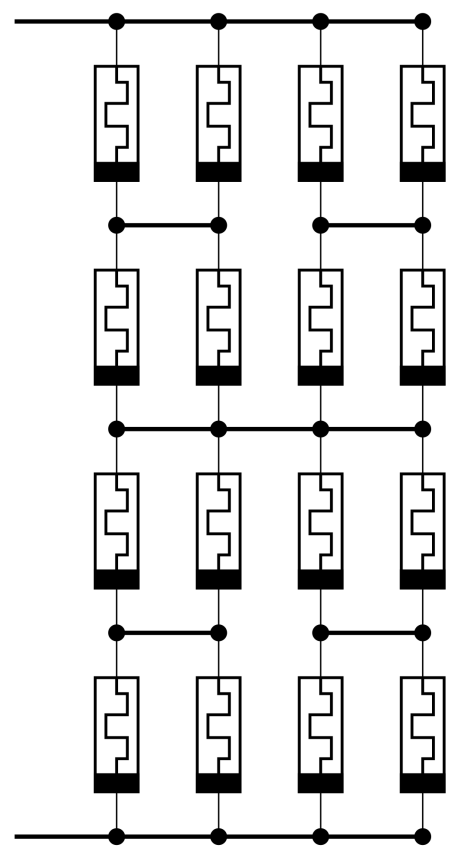

(a)

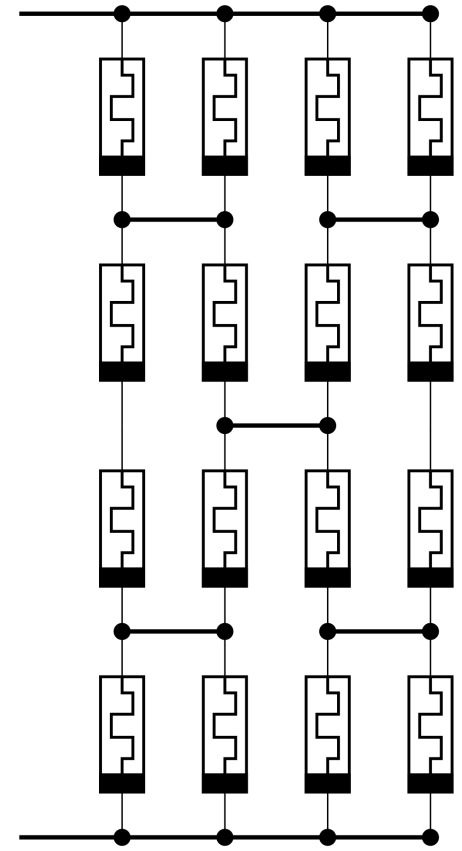

(b)

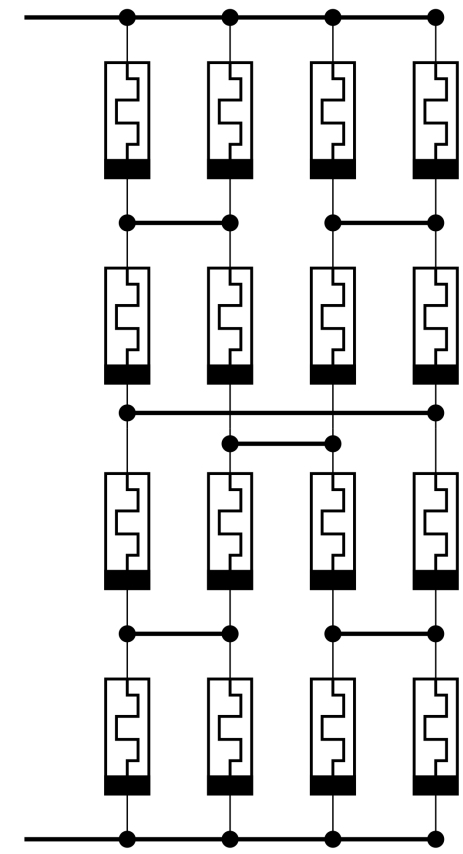

(c)

Figure 2. Measured general circuits. (a) H-fractal type of array. (b) Checkerboard type of array. (c) Our newly introduced array.

In this article a third general circuit design is proposed (Figure 2c), which can be implemented as a $2 \times 2 \times 4$, three-dimensional grid structure on a multilayer carrier. A structure proposal can be seen in Figure 3a. This new circuit had a better compromise between open and short connection faults, but can only be constructed effectively in a three dimensional structure. The disadvantage is that since 
the height of the grid was even, and the top and bottom electrodes are aligned, they cannot form a crossbar network.

A workaround could be that this type of network can scale with the height of the $2 \times 2$ column, and it can be $2 \times 2 \times 3$ or $2 \times 2 \times 5$ sized. These new non-general networks result in different memristor parameters. The advantage of an odd height is that it can be realized in a crossbar network as it can be seen in Figure $3 b$.

Memristor networks that use binary memristors as building components will technically result in a discrete memory capacity as either component can be in the OFF or ON state. The overall resistance value can be calculated for every combination, which is a limited number of possible resistances. However, with sufficiently large grids, this effect can be neglected as the individual operational variances of the elements are also summing up, resulting in a complex macro-characteristics.

Another important property to consider is the used chip area. These networks should be implemented efficiently on a chip as a two dimensional crossbar network. The implementation of the previous networks was only possible using sixteen times more chip area for the emulation of a single device. The new network uses only four times more area with a similar reliability gain, as compared to a single memristor.

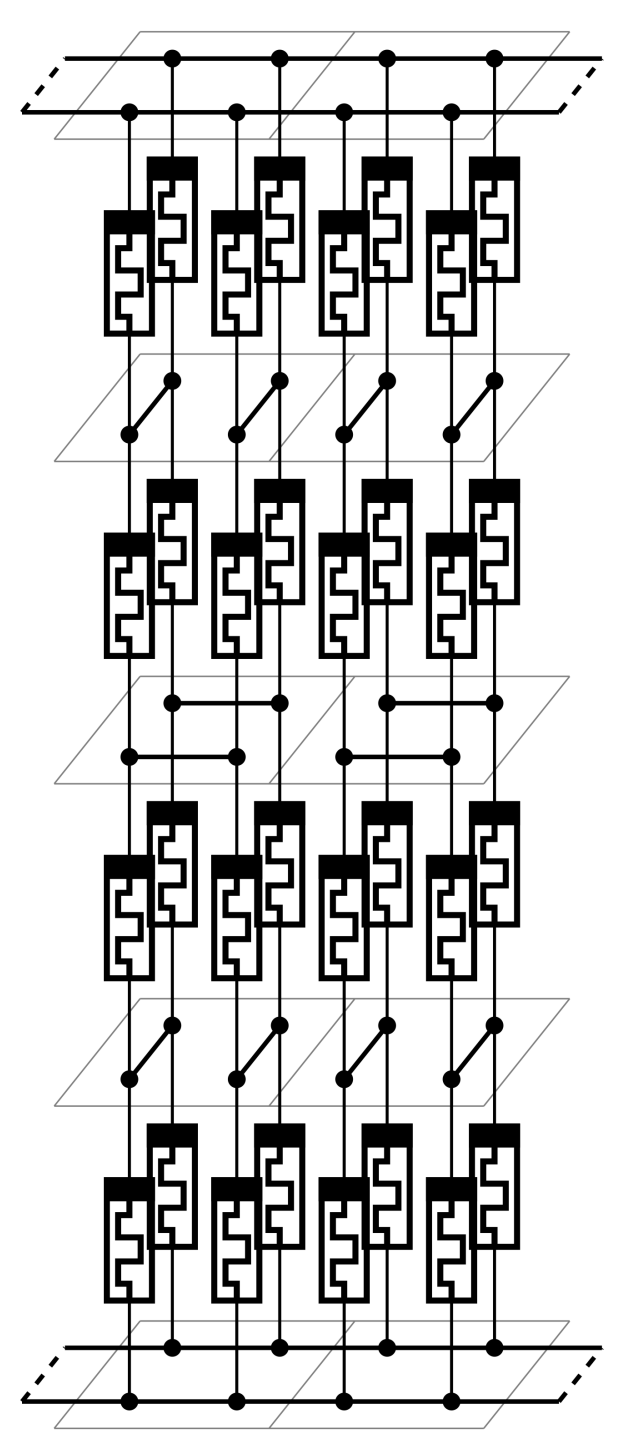

(a)

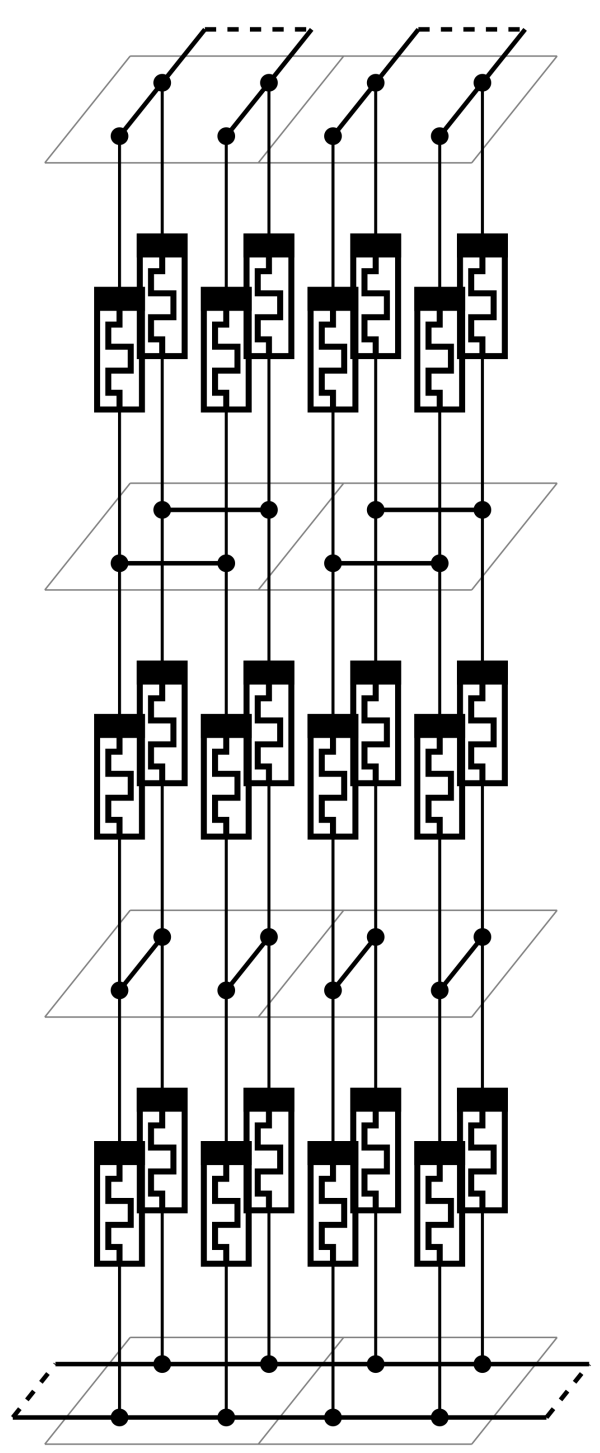

(b)

Figure 3. The proposed three dimensional cell structures. (a) Two emulated cells from a $2 \times 2 \times 4$ array. (b) Two emulated cells from a $2 \times 2 \times 3$ array. 


\section{Results}

\subsection{Single Memristor Measurements}

First test measurements were prepared with a single memristor device. Here two types of signals were used. The first one was a single, $2.4 \mathrm{~s}$ long $2.5 \mathrm{~V}$ writing pulse, which shows some parameters of the device. The results can be seen in Figure 4. The average ON state was $57 \mathrm{k} \Omega$, the average OFF state was $11.5 \mathrm{M} \Omega$. The ON/OFF ratio is approximately 200.

The second type of signal is a sequence of a writing and an erasing signal. The writing pulse was $160 \mathrm{~ms}$ long, while the erasing one was shorter, $40 \mathrm{~ms}$. The results can be seen in Figure 5 . The writing process was faster and starts at a lower voltage level, but the switching was not as sharp as in the previous case (Figure 4). During the reading sequence, the small amplitude pulses did not change the state of the memristor.

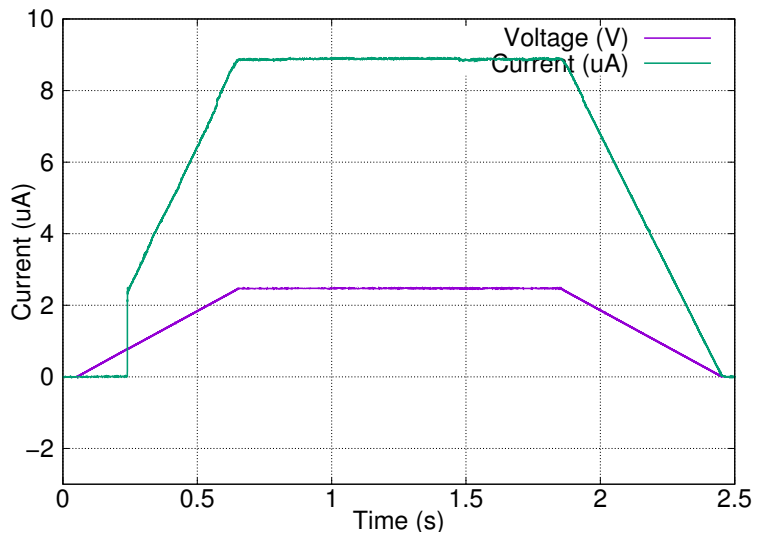

(a)

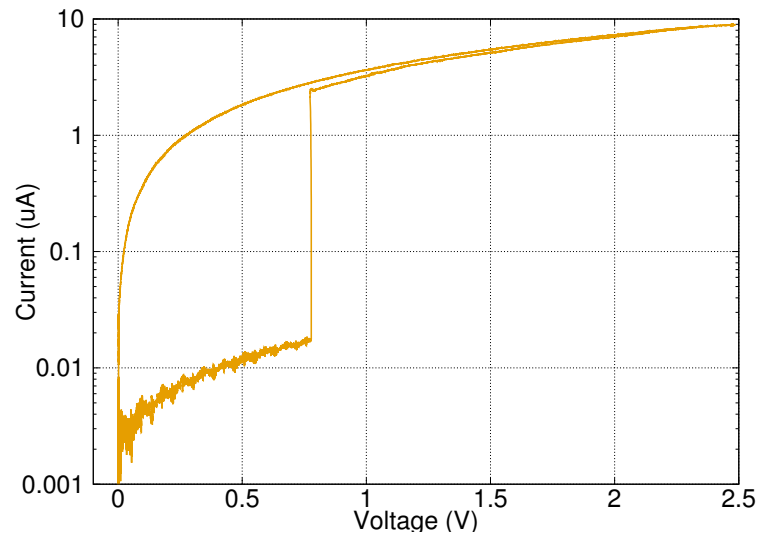

(b)

Figure 4. Long timescale measurement on a single memristor device with focus on the writing characteristics. (a) The input signal and output response in the time domain. (b) Phase portrait of the measurement. Switching is very sharp and the ON/OFF ratio is at least 100.

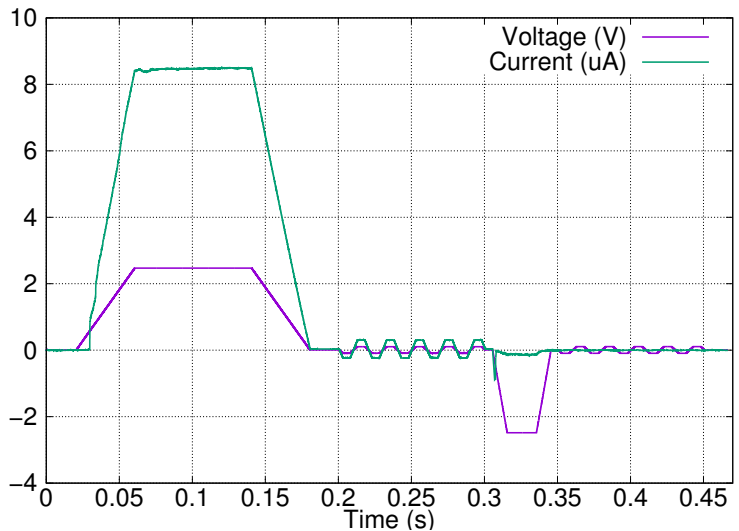

(a)

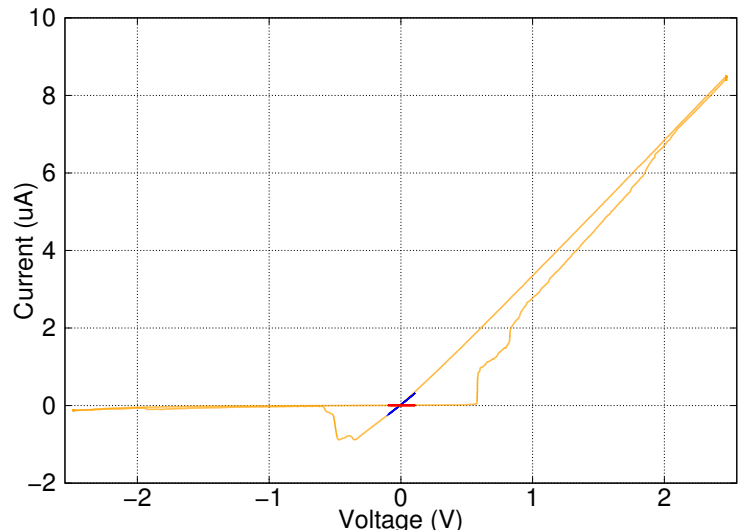

(b)

Figure 5. Write-read-erase-read cycle measurement on a single memristor device. (a) The input signal and output response in the time domain. (b) Phase portrait of the measurement. The read sequence after the write and erase pulses are colored as blue and red, respectively.

\subsection{Memristor Emulation Comparison Measurements}

The following measurements were carried out on four different network types and on a single memristor for reference. The measuring signal is alternating write-erase sinusoidal pulses with a length of $23 \mathrm{~ms}$ in a sequence of 50 cycles. 
This measurement is supposed to simulate a general training scenario, where an analogue memristor characteristic is expected and the training is done by several small pulses. According to this consideration, the writing pulses of the measurement have not enough energy to change the state of a single memristor into its $\mathrm{ON}$ state.

The results can be seen in Figures 6 and 7. Subfigures (a),(c) and (e) show the voltage-current diagram of the whole signal. Subfigures $(\mathbf{b}),(\mathbf{d})$ and $(\mathbf{f})$ are the voltage-current diagrams of the average of all 50 write-erase signals with the current shown on a logarithmic scale.

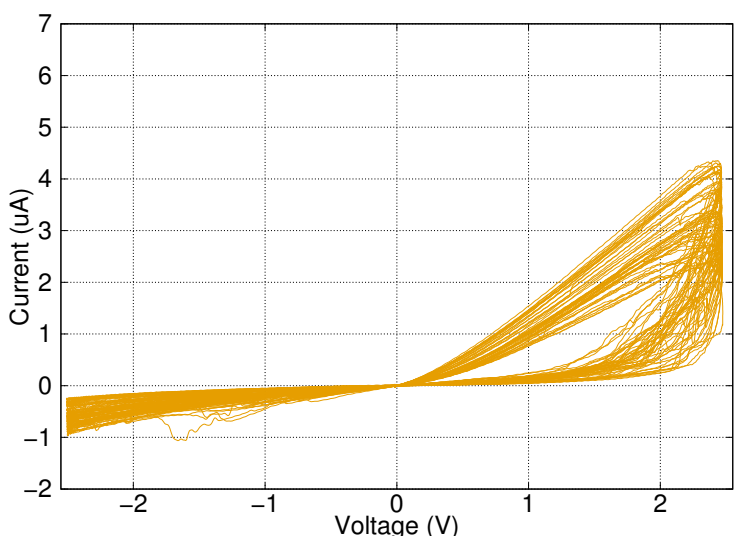

(a)

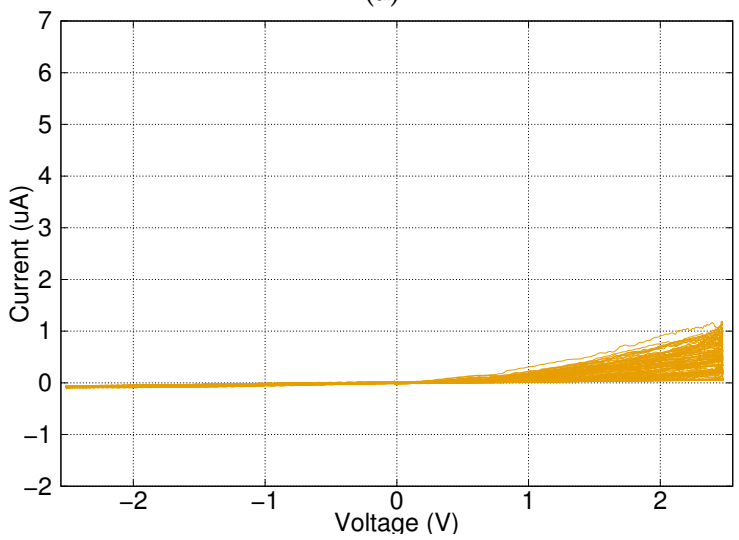

(c)

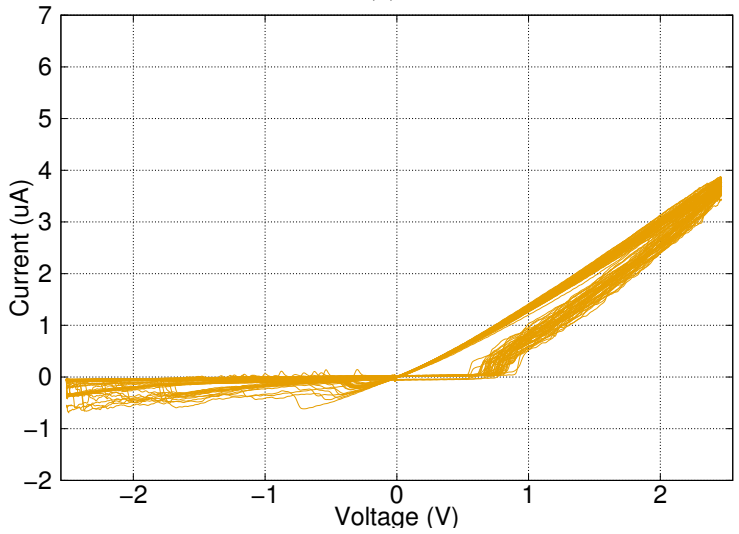

(e)

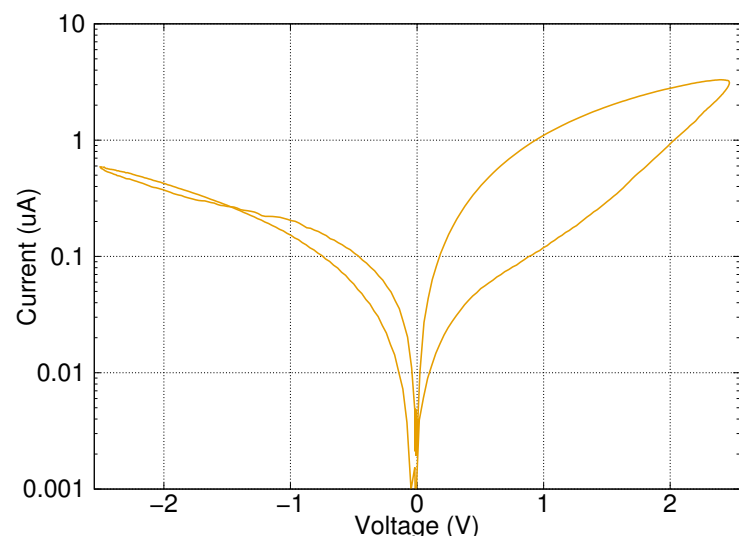

(b)

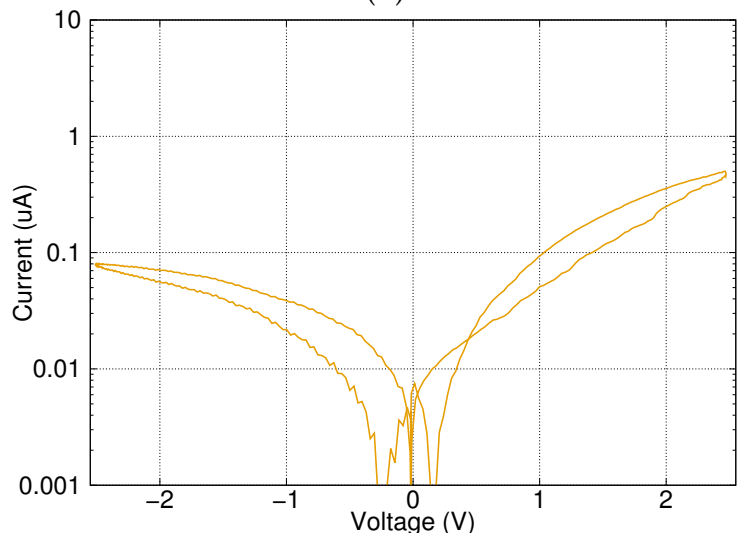

(d)

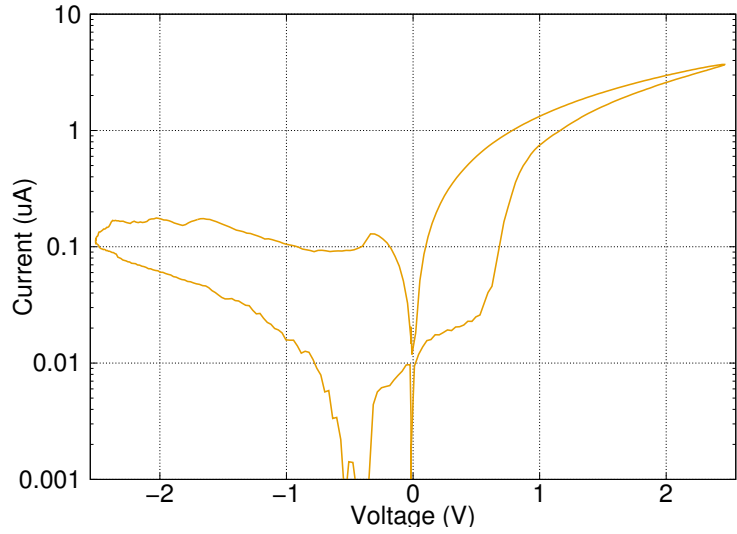

(f)

Figure 6. Short-time pulses on a single memristor, the checkerboard like and the H-fractal memristor network, respectively. $(\mathbf{a}, \mathbf{c})$ and $(\mathbf{e})$ show the voltage-current diagram of the whole signal. $(\mathbf{b}, \mathbf{d}, \mathbf{f})$ are the average of all 50 write-erase signals on a logarithmic scale. 


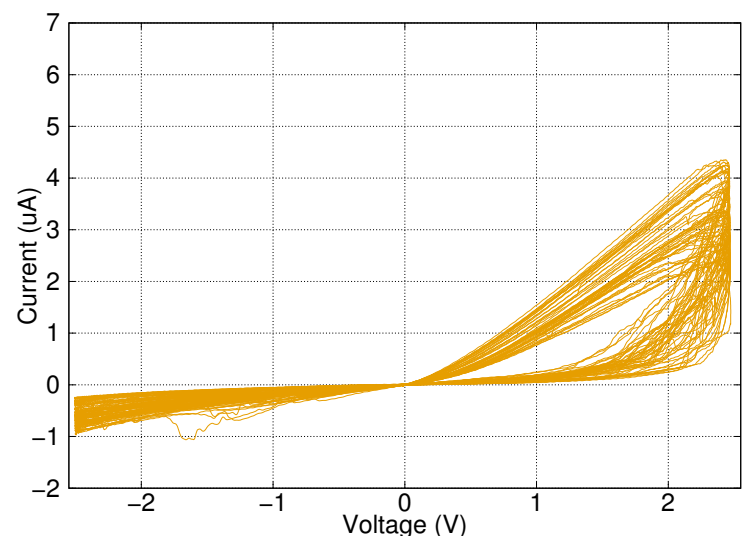

(a)

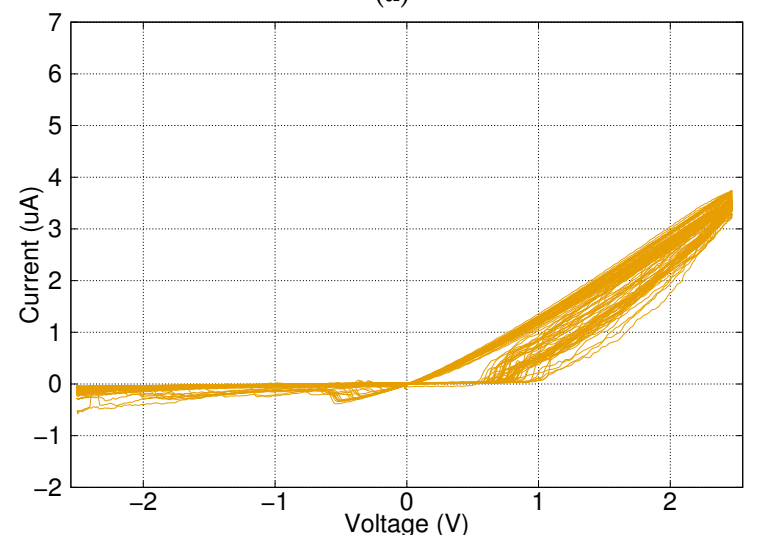

(c)

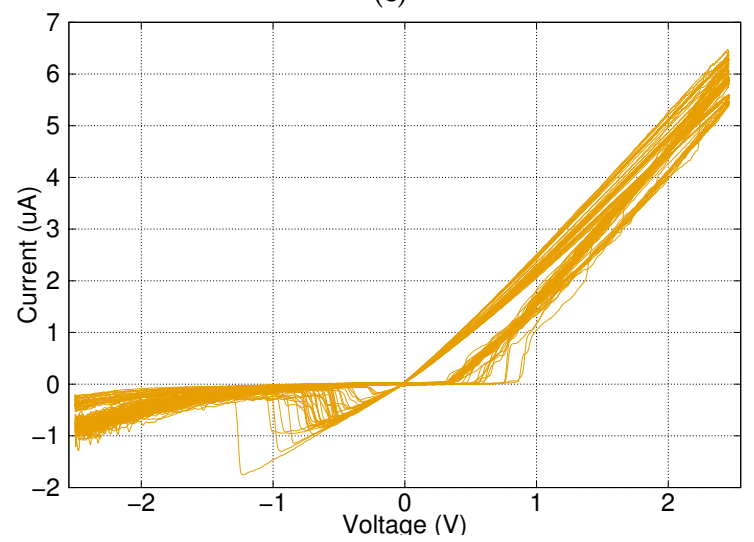

(e)

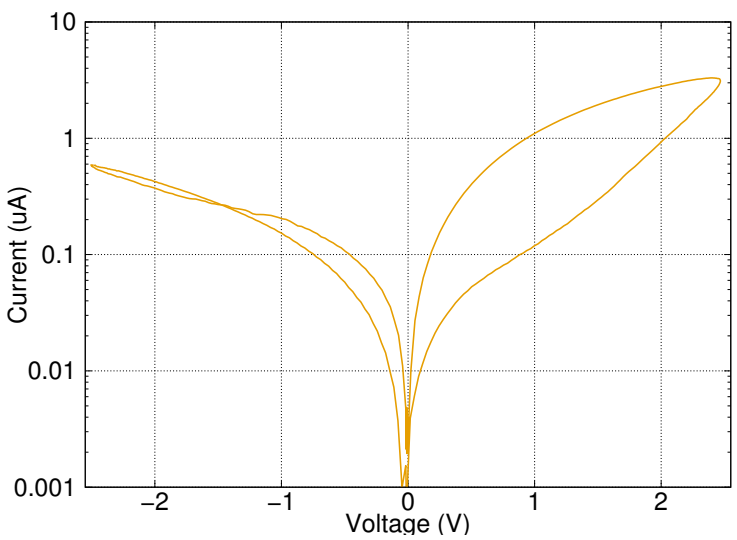

(b)

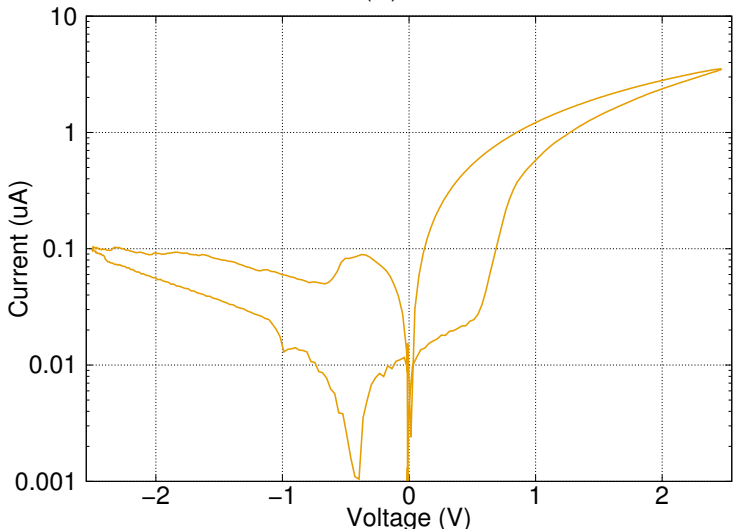

(d)

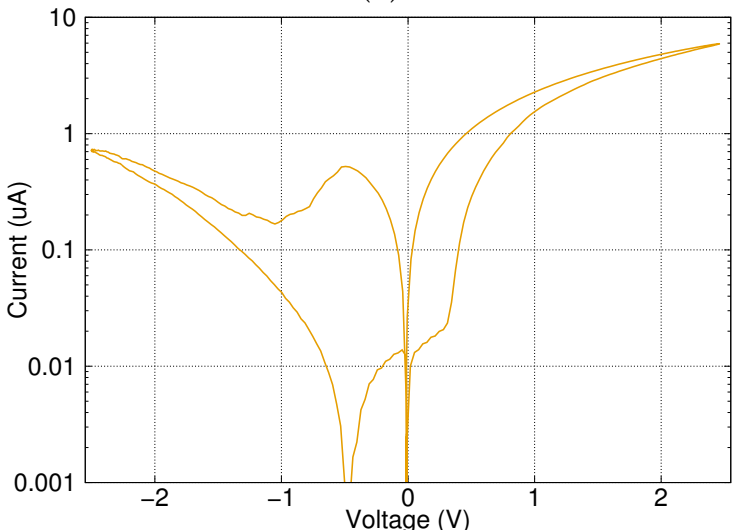

(f)

Figure 7. Short-time pulses on a single memristor, the new three dimensional network with sixteen memristors and the reduced network with twelve memristors, respectively. (a,c) and (e) show the voltage-current diagram of the whole signal. $(\mathbf{b}, \mathbf{d})$ and $(\mathbf{f})$ are the average of all 50 write-erase signals on a logarithmic scale.

\section{Discussion}

The checkerboard type of network was practically unable to switch its state significantly compared to other solutions. This was probably due to the limited number of parallel connections in the network, which produced less possible routes to open. Higher control voltage could change its state, but the risk of device damage increases with the increased after-switch current. Longer pulses could also help, but it makes the writing process slower. 
The results for the $\mathrm{H}$-fractal type of network are very similar to the newly introduced network regarding the writing of the state into low resistance position. However, this type of network has problems with erasing the state into the OFF state and could get stuck at an in-between state.

The ON and OFF resistance values of the network with twelve memristors are lower than the other networks due to the reduced number of serial layers. However, when one compares it to a single memristor, it has lower ON resistance value and higher OFF resistance, meaning the network is more sensitive to control signals than only one memristor. In other words, a pulse with the same voltage level could make a clearer distinction between the initial and after states.

The previous simulation results suggested that the switching speed could decrease using memristor grids. Surprisingly, the switching speed did not decrease, but increased instead. The networks are approximately three times faster than a single memristor. This is fairly unexpected, as the control voltage stayed constant in both measurements, which means that the voltage on any single memristor in a network measurement had to be strictly lower than in the case of a single device measurement at any given time during measuring.

One explanation of this phenomenon could be the following: under the threshold voltage, the device behaves as a very small capacitor. As the metal flows into the dielectric matter to build up the filament, the partially charged capacitor discharges, causing a short-time high-energy electric current burst. The other devices are sensitive to fast current changes and the filament forming is starting in them as well. It can be seen as a "domino effect" with the consecutive memristors. If any of the OFF state memristors in a series switches to the ON state, the rest will automatically switch as well immediately after.

If any of the memristors which closes the source in the series, opens, the rest will automatically open immediately after.

Based on the above presented measurements the following parameter values were acquired, presented in Table 1. The resistance values are the average ON/OFF ratio values of the 50 cycle long measurement sequence.

Table 1. The table shows the main properties of emulating memristor networks. Higher ON/OFF ratio is considered better and the best values are indicated accordingly, namely the highest OFF resistance, the lowest $\mathrm{ON}$ resistance and the highest overall ON/OFF ratio. Lower dispersion is also considered better. The lowest is indicated.

\begin{tabular}{ccccc}
\hline Measured Object & OFF Resistance & ON Resistance & ON/OFF Ratio & Dispersion Index \\
\hline Single memristor & $5.7889 \mathrm{M} \Omega$ & $0.7185 \mathrm{M} \Omega$ & 8.0569 & 0.04553 \\
H-fractal network & $19.472 \mathrm{M} \Omega$ & $0.6717 \mathrm{M} \Omega$ & 28.990 & 0.02718 \\
Checkerboard network & $20.322 \mathrm{M} \Omega$ & $5.4633 \mathrm{M} \Omega$ & 3.7197 & 0.04921 \\
3D $2 \times 2 \times 4$ network & $\mathbf{2 0 . 6 5 1} \mathrm{M} \Omega$ & $0.7072 \mathrm{M} \Omega$ & $\mathbf{2 9 . 2 0 1}$ & $\mathbf{0 . 0 1 8 0 0}$ \\
3D $2 \times 2 \times 3$ network & $9.3426 \mathrm{M} \Omega$ & $\mathbf{0 . 4 1 9 4} \mathbf{M} \Omega$ & 22.276 & 0.02491 \\
\hline
\end{tabular}

Another important feature of th networks to note is the stronger nanobattery effect [16]. This causes the visible shift of the zero current level after the erasing pulse. The nanobattery effect is undesired in most applications, but can be dealt with by an appropriate control voltage and timing. It can also be taken advantage of, in some scenarios.

\section{Conclusions}

Two new types of memristor networks have been introduced, which are able to emulate more reliable memristors. Measurements have been successfully carried out for both the previously presented networks and the new networks. The measurements provided new information about the macro-characteristics of memristor networks compared to the previous simulations. The increased switching speed of memristor networks should be further investigated. This solution can be used with existing devices to support the implementation of neuromorphic applications. 
Author Contributions: Conceptualization, Á.R. and G.C.; methodology, D.H.; software, D.H.; validation, D.H., Á.R. and G.C.; formal analysis, Á.R.; investigation, Á.R.; resources, G.C.; data curation, D.H.; writing-original draft preparation, D.H.; writing—review and editing, Á.R. and G.C.; visualization, D.H.; supervision, G.C.; project administration, G.C.; funding acquisition, G.C.

Funding: This research was funded by the Hungarian Government grant number 2018-1.2.1-NKP-00008: Exploring the Mathematical Foundations of Artificial Intelligence. The Application Process Charge was funded by KAP19-1.1-ITK of the Pazmany Peter Catholic University

Acknowledgments: The authors gratefully acknowledge the support of grant 2018-1.2.1-NKP-00008 of the Hungarian National Research, Development and Innovation Office (NKFIH), the fund of KAP19-1.1-ITK of the Pazmany Peter Catholic University and the support of the Roska Tamás Doctoral School of Science and Technology.

Conflicts of Interest: The authors declare no conflict of interest. The funders had no role in the design of the study; in the collection, analyses, or interpretation of data; in the writing of the manuscript, or in the decision to publish the results.

\section{Abbreviations}

The following abbreviations are used in this manuscript:

VLSI Very large scale integration

APM Analog purpose memristor

DPM Digital (or discrete) purpose memristor

BPM Binary purpose memristor

CMOS Complementary metal-oxide-semiconductor

RAM Random access memory

FPGA Field programmable gate array

\section{References}

1. Chua, L. Memristor-the missing circuit element. IEEE Trans. Circuit Theory 1971, 18, 507-519. [CrossRef]

2. Strukov, D.B.; Snider, G.S.; Stewart, D.R.; Williams, R.S. The missing memristor found. Nature 2008, 453, 80. [CrossRef] [PubMed]

3. Vaidyanathan, S.; Volos, C. Advances in Memristors, Memristive Devices and Systems; Springer: Berlin/Heidelberg, Germany, 2017; Volume 701.

4. Wang, Z.; Joshi, S.; Savel'ev, S.E.; Jiang, H.; Midya, R.; Lin, P.; Hu, M.; Ge, N.; Strachan, J.P.; Li, Z.; et al. Memristors with diffusive dynamics as synaptic emulators for neuromorphic computing. Nat. Mater. 2017, 16, 101. [CrossRef] [PubMed]

5. Indiveri, G.; Linares-Barranco, B.; Legenstein, R.; Deligeorgis, G.; Prodromakis, T. Integration of nanoscale memristor synapses in neuromorphic computing architectures. Nanotechnology 2013, 24, 384010. [CrossRef] [PubMed]

6. Magyari-Köpe, B.; Song, Y.; Duncan, D.; Zhao, L.; Nishi, Y. Research Update: Ab initio study on resistive memory device optimization trends: Dopant segregation effects and data retention in $\mathrm{HfO}_{2-x}$. APL Mater. 2018, 6, 058102. [CrossRef]

7. Weste, N.H.; Eshraghian, K. Principles of CMOS VLSI design: A systems perspective. NASA STI/Recon Tech. Rep. A 1985, 85554.

8. Lewis, D.L.; Lee, H.H.S. Architectural evaluation of 3D stacked RRAM caches. In Proceedings of the IEEE International Conference on 3D System Integration, San Francisco, CA, USA, 28-30 September 2009; pp. 1-4.

9. Cong, J.; Xiao, B. mrFPGA: A novel FPGA architecture with memristor-based reconfiguration. In Proceedings of the IEEE/ACM International Symposium on Nanoscale Architectures, Washington, DC, USA, 8-9 June 2011; pp. 1-8.

10. Knowm Inc. Homepage. Available online: https:// knowm.org (accessed on 31 March 2019).

11. Stathopoulos, S.; Khiat, A.; Trapatseli, M.; Cortese, S.; Serb, A.; Valov, I.; Prodromakis, T. Multibit memory operation of metal-oxide bi-layer memristors. Sci. Rep. 2017, 7, 17532. [CrossRef] [PubMed]

12. Di Ventra, M.; Pershin, Y.V. On the physical properties of memristive, memcapacitive and meminductive systems. Nanotechnology 2013, 24, 255201. [CrossRef] [PubMed] 
13. Xu, R.; Jang, H.; Lee, M.H.; Amanov, D.; Cho, Y.; Kim, H.; Park, S.; Shin, H.J.; Ham, D. Vertical MoS2 double layer memristor with electrochemical metallization as an atomic-scale synapse with switching thresholds approaching $100 \mathrm{mV}$. Nano Lett. 2019, doi:10.1021/acs.nanolett.8b05140. [CrossRef] [PubMed]

14. Rák, Á.; Cserey, G. Emulation of analog memristors using low yield digital switching memristors. In Proceedings of the European Conference on Circuit Theory and Design (ECCTD), Dresden, Germany, 8-12 September 2013; pp. 1-4.

15. Lanza, M.; Wong, H.S.P.; Pop, E.; Ielmini, D.; Strukov, D.; Regan, B.C.; Larcher, L.; Villena, M.A.; Yang, J.J.; Goux, L.; et al. Recommended methods to study resistive switching devices. Adv. Electron. Mater. 2019, 5, 1800143. [CrossRef]

16. Valov, I.; Linn, E.; Tappertzhofen, S.; Schmelzer, S.; van den Hurk, J.; Lentz, F.; Waser, R. Nanobatteries in redox-based resistive switches require extension of memristor theory. Nat. Commun. 2013, 4, 1771. [CrossRef] [PubMed]

(C) 2019 by the authors. Licensee MDPI, Basel, Switzerland. This article is an open access article distributed under the terms and conditions of the Creative Commons Attribution (CC BY) license (http:/ / creativecommons.org/licenses/by/4.0/). 